\title{
McDowell y el realismo ingenuo. Mind and World ante el argumento por la ilusión ${ }^{1}$
}

\author{
McDowell and Naïve realism. \\ Mind and World facing the argument from illusion
}

Juan Manuel Saharrea ${ }^{2}$

CONICET / Universidad Nacional de Córdoba, Argentina

Recepción: 14 de septiembre del 2020

Evaluación: 05 de marzo del 2021

Aceptación: 26 de marzo del 2021

\footnotetext{
1 El artículo se deriva del plan de trabajo de la beca doctoral (2015-2020) "La naturaleza del contenido perceptivo para determinar el rol justificatorio de la experiencia”, subvencionada por el Consejo Nacional de Investigaciones Científicas y Técnicas (CONICET), y enmarcada en el grupo de Investigación “Comprender y significar. Dificultades de aprendizaje y neuroeducación”, Proyecto Consolidar I, acreditación y subsidio: Secretaría de Ciencia y Técnica, Universidad Nacional de Córdoba, Res. Secyt-UNC N ${ }^{\circ} 411 / 18,455 / 18$ y 472, con filiación en el Centro de Investigaciones Maria Saleme de Burnichón, Facultad de Filosofía y Humanidades de la UNC.

2 Doctor en Filosofía. Instituto de Investigaciones Psicológicas (CONICET / Universidad Nacional de Córdoba).

Correo electrónico: jsaharrea@mi.unc.edu.ar
} 


\title{
Resumen
}

Mediante el análisis conceptual el artículo reconstruye algunos aspectos de Mind and World de John McDowell, orientados a ampliar el rol explicativo de la tesis de la "carencia de límites de lo conceptual". Para esto se vincula dicha tesis, que corre el riesgo de ser objeto de la objeción de idealismo en sus variantes "más perniciosas", con la idea de percepción como "apertura al mundo", que niega decididamente un idealismo semejante. Si bien la defensa del rol justificatorio del contenido perceptivo da cuenta de esta última tesis, no muestra, al menos explícitamente, relaciones posibles con la primera. Este artículo, en cambio, propone resolver esta tensión. Así reconstruye la posible respuesta del conceptualismo de McDowell al argumento por la ilusión. De este modo, a partir de la distinción entre posibilidad ordinaria de error perceptual y posibilidad en principio de error perceptual -derivada del tratamiento de McDowell sobre la incorregibilidad perceptual- se concluye que la "carencia de límites de lo conceptual" y la afirmación de que ordinariamente la percepción nos proporciona conocimiento directo de hechos del mundo, no son incompatibles desde la perspectiva de la posibilidad ordinaria.

Palabras clave: McDowell, percepción, posibilidad, argumento, ilusión.

\begin{abstract}
This article rebuilds, through conceptual analysis, some of the aspects in Mind and World of John McDowell aimed at broadening the explanatory role of "the unboundedness of the conceptual" thesis. In order to achieve this, the aforesaid thesis - which runs the risk of being object of the idealism's objection in its "most pernicious" variants has been connected with the idea of perception as "openness to the world"- which decidedly denies a similar idealism. Although the defense of the perceptual content's justifying role acknowledges this last thesis, it does not show possible connections with the first one (at least, not in an explicit way). This article, on the contrary, aims at easing this tension, thereby rebuilding McDowell conceptualism's answer to the argument from illusion. Thus, the conclusion drawn from the distinction between the ordinary possibility of the perceptual error and possibility in principle (of perceptual error) -derived from McDowell's treatment of perceptual incorrigibility- is that "the unboundedness of the conceptual" and the assertion that perception provides us with direct knowledge of the world's facts are not incompatible under the perspective of the ordinary possibility.
\end{abstract}

Keywords: McDowell, perception, possibility, argument, illusion. 


\section{Un realismo ingenuo que reúna la mente y el mundo}

En el presente trabajo vinculamos la defensa del conceptualismo de John McDowell con el realismo ingenuo. Sostendremos que si el conceptualismo defendido por el filósofo de Pittsburgh en Mind and World no da cuenta de los casos de ilusión perceptiva, la idea de la "carencia de límites de lo conceptual" -central en dicha obra- resulta susceptible de una objeción de idealismo en sus variantes más perniciosas. Mostraremos el impacto de esta concepción para la defensa del conceptualismo, en especial para dar sustento a la idea de experiencia perceptiva como "apertura al mundo" (McDowell,1994/6, p. 26) en la que tanto insiste McDowell.

El trabajo se organiza de la siguiente manera. En la sección 2 presentamos dos líneas de argumentación sobre la percepción: el conceptualismo y el realismo ingenuo, que son consideradas por McDowell de manera independiente. En la sección 3 delimitamos los escollos que debe enfrentar el conceptualismo. En la cuarta sección exponemos la tesis de la carencia de límites de lo conceptual. En esta fase mostramos que la concepción de la experiencia como apertura al mundo se robustece notablemente si se analiza, a partir de ella, el argumento por la ilusión. Señalamos, asimismo, que McDowell brinda inicialmente algunos elementos para responder a dicha objeción sin mayor desarrollo. En esta medida, profundizamos la distinción entre 'posibilidad ordinaria de error perceptivo o factual' y 'posibilidad en principio' (las expresiones son nuestras). Finalmente mostramos cómo esta comprensión del error perceptivo incide en la comprensión de la carencia de límites de lo conceptual. Ambos elementos, por último, convergen para respaldar la idea de experiencia como apertura al 'diseño de la realidad' o 'apertura al mundo' (pp. 26-27).

\section{Dos líneas de argumentación sobre la percepción}

Desde hace más de dos décadas John McDowell lleva adelante la defensa de la "plausibilidad de un empirismo mínimo" (p. i $)^{3}$. En su célebre obra Mind and World (1994/6) desarrolla esta propuesta epistemológica que ubica a la experiencia como origen y fundamento de todas nuestras creencias acerca del

\footnotetext{
3 Mantenemos el sistema de numeración i, ii iii, presente en la fuente, para referenciar la introducción del libro. Empleamos el sistema arábigo para referenciar el cuerpo del texto. A excepción de que lo aclaremos, seguimos la traducción de Miguel Ángel Quintana Paz -única disponible al españolde Mente y Mundo.
} 
mundo empírico. La plausibilidad de este empirismo depende básicamente de dos cuestiones fundamentales: en primer lugar, entiende que la experiencia juega efectivamente un rol epistémico en los procesos de justificación de nuestras creencias. Las experiencias, por tanto, justifican nuestros juicios; son razones para creer que éste es el caso ${ }^{4}$. En segundo lugar, la mera posibilidad de que nuestros pensamientos tengan contenido, es decir, que sean sobre algo, radica en el hecho de que estos deben ser responsables ante la experiencia ( $\mathrm{p}$. 18), que se constituye como un tribunal. Tanto el contenido de una creencia como el de la percepción -el hecho de que seamos capaces de representar el mundo correcta e incorrectamente- se funda en la normatividad propia de los vínculos entre la mente y el mundo.

Como resultado de esta argumentación, McDowell (1994/6) señala que cuando percibimos, nuestra experiencia "capta" rasgos del mundo. Esta concepción en torno a la percepción al modo de "apertura al diseño (layout) de la realidad" o simplemente como 'apertura al mundo' puede ubicarse en el marco de la defensa de un realismo ingenuo, conforme al cual la percepción accede directamente al conocimiento de objetos del mundo (p. 26).

En la actualidad el realismo ingenuo es defendido desde dos vertientes diferentes: por un lado, John Searle (2015), por el otro, John Fish (2009) y Mike Martin (2002). Los tres sostienen que la experiencia verídica se pone en contacto de manera inmediata con los objetos del mundo físico. Sin embargo, mientras los dos últimos defienden que ésta no tiene un contenido representacional, Searle (2015) no niega que lo tenga; es más, le parece bastante incomprensible que pueda negarse este rasgo. Este considera, a diferencia de aquellos, que la premisa de que asumir el contenido representacional de la experiencia podría favorecer el rechazo del realismo ingenuo o directo, es absolutamente inaceptable.

Esta posición podría acercarlo a la concepción de McDowell, pero Searle se diferencia de McDowell porque éste acepta dicha premisa como parte de una objeción atendible y, sobre la base de su aceptación, intenta defender el realismo directo. Él en cambio defiende el realismo ingenuo rechazando dicha objeción al considerarla parte de un "mal argumento" (Searle, 2015, pp. 23-27) e incluso defendiendo que el hecho de que una percepción verídica y una ilusoria compartan contenido no dice nada en contra del realismo

${ }^{4}$ Empleamos ‘juicio’ y ‘creencia’ como sinónimos. 
ingenuo, toda vez que, por cierto, se atiende al vocabulario para el análisis del contenido perceptivo que él propone.

El punto que nos interesa rescatar de Fish y Martin, sin embargo, es que, si bien hay diferencias internas entre ambos filósofos, sus compromisos fundamentales señalan, en el debate actual, la pervivencia de la ligazón tradicional entre, por un lado, la atribución de contenido representacional a la experiencia, y las dificultades en la defensa del realismo ingenuo, por otro. De hecho, tal ccomo lo reconoce Fish (2009) "(...) en la filosofía reciente, la afirmación de que la experiencia tiene 'contenido' ha sido entrelazada profundamente a la idea de que este contenido es algo común a experiencias verídicas y no verídicas" (p. 7). Por lo que en rigor alguien que, como McDowell, sostiene que la experiencia tiene contenido, estaría en una posición débil 5 .

Esto es cierto. Sin embargo, hay un aspecto del realismo ingenuo de índole más general que puede rescatarse del modo en que lo delimitara hasta el final de su vida Hilary Putnam. Putnam $(1997 ; 2012)$ hace hincapié en el contraste entre realismo ingenuo y teorías de los datos sensoriales, argumentando que la postulación de "interfaces epistémicas" entre la mente y el mundo es un paso problemático para la reflexión epistemológica. Ahora bien, esto en nada cuestiona que pueda o no atribuirse un contenido a la experiencia. Sin desestimar este aspecto de la discusión, Putnam cree que hay algo importante en no conceder que el error perceptivo sea lo que los detractores del realismo ingenuo suponen. Esos detractores a veces cobran la forma de un escéptico radical y otras no. En cualquier caso, en este trance de la delimitación de la percepción hay algo importante para considerar.

Siguiendo la reconstrucción del realismo ingenuo de McDowell, uno de nuestros intereses es señalar que en Mind and World hay elementos que contribuyen a la defensa de un realismo ingenuo sin cuestionar, a diferencia de Fish y de Martin, que la experiencia tenga contenido. Abordar el contraste, sin embargo, entre los actuales defensores del realismo ingenuo y la posición de McDowell, supera los límites del presente artículo. Entendemos que Fish y de Martin presentan puntos de acuerdo con McDowell en al menos dos cuestiones: 1) el rechazo del argumento por la ilusión con base en la

5 Discutir aspectos más específicos de su propuesta, entre ellos: su interesante y complejo vocabulario para analizar la fenomenología de la percepción y su crítica del disjuntivismo, uno de cuyos defensores más reconocidos es McDowell, excede los límites de este artículo. 
consideración del error perceptivo; 2) la necesidad de despejar obstáculos para la defensa del realismo ingenuo neutralizando un escepticismo extremo o radical.

Bajo la reconstrucción de Putnam, el realismo ingenuo (o directo) se opone a visiones tradicionales de la percepción, que postulan interfaces epistémicas entre la experiencia y el mundo físico. Las teorías que plantean datos sensoriales se ubican justamente en esta línea; desde el empirismo clásico (cuyos representantes hablaban en términos de "impresiones" o "ideas") hasta los empiristas lógicos del siglo XX (Putnam, 1997, p. 123). De acuerdo con estas teorías de interfaces epistémicas, dado que la experiencia ofrece casos de percepción no verídica-debido a que es falible-, entonces no percibimos directamente el mundo. La ventaja explicativa más evidente de los detractores del realismo ingenuo es la posibilidad de dar cuenta de la falibilidad de la percepción a partir de la postulación de intermediarios epistémicos. En este sentido, los casos falibles demuestran que accedemos al mundo sólo a través de interfaces (por ejemplo, datos sensoriales, impresiones o ideas).

El realismo ingenuo, a la luz de esta objeción, queda bastante exigido. Para ser mínimamente sustentable, más allá de su correspondencia con nuestra fenomenología ordinaria, debería dar alguna respuesta a la falibilidad de la percepción, es decir, a los casos de error perceptivo. En tal medida resulta ineludible que discuta el argumento por la ilusión, el cual decididamente resulta ser el arma principal para desestimar el realismo ingenuo, al punto de que durante muchos siglos -según Putnam- se ha dado por descontada su validez para la reflexión epistemológica $(1997 ; 2012)$. Este argumento lleva al extremo la cuestión de la falibilidad de la experiencia.

No hay una variante del argumento por la ilusión compartida por toda la tradición. De acuerdo a la versión de Putnam consta, en su estructura más simple, de dos premisas y conclusión: 1) son posibles las experiencias ilusorias; 2) nuestras experiencias verídicas e ilusorias son cualitativamente idénticas, por tanto, 3) no percibimos objetos del mundo, sino interfaces epistémicas. La conclusión supone que las experiencias ilusorias son aquellas en donde nuestra experiencia no accede a objetos del mundo físico. Complementariamente las experiencias verídicas son aquellas que sí acceden a objetos del mundo físico (Putnam, 1997, pp. 27-52).

La oposición entre realismo directo o ingenuo y teorías que plantean interfaces epistémicas, sin embargo, no constituye un elemento importante en la defensa del empirismo mínimo. Las razones de ello no son fruto de una 
falta de interés o estimación por parte de McDowell acerca de la problemática. En artículos previos y contemporáneos a su obra magna, aborda la defensa de un realismo directo. Probablemente "Knowledge and the internal" (1995) sea el caso más característico de defensa del realismo ingenuo. Su estrategia allí, sin embargo, es considerar el argumento por la ilusión como una "interiorización" de la idea de "espacio lógico de las razones" (McDowell, 1998, p. 395). Su argumentación apunta, en este sentido, a exteriorizar la idea de espacio lógico de las razones y descartar otras motivaciones para considerar lo mental como algo interno. No obstante, en el contexto de la defensa del empirismo mínimo no menciona la necesidad de defender dicho realismo ni, complementariamente, cuestionar las teorías de interfaces epistémicas.

Hilary Putnam (Smith, 2002, p. 213) argumentó en dos oportunidades, con cierto asombro, que la postura de Mind and World resultaría más comprensible si se ubicase en la línea de la defensa del realismo directo que McDowell aborda de manera paralela al modo de una concepción 'disjuntivista' de la experiencia, según la cual entre una percepción verídica e ilusoria no hay un factor común denominador (por ejemplo, la negación de la premisa (2) del párrafo anteriormente citado $)^{6}$. McDowell, en su contrarréplica, descarta de plano esta interpretación. Insiste en que -como veremos en la sección 1- los únicos escollos que debe superar su empirismo mínimo son el coherentismo epistemológico y el Mito de lo Dado:

Putnam evidentemente piensa que mi objetivo principal es insistir en el "realismo directo" sobre la percepción, rechazar una imagen en la que la experiencia perceptiva hace contacto con el mundo solo a través de una interfaz (at a interface). Habla como si esta concepción de la interfaz simplemente estuviera allí como un problema para nosotros, debido a cómo se ha desarrollado la filosofía moderna. Para defender el "realismo directo" en este tipo de espíritu, habría que socavar todas las racionalizaciones que los filósofos han inventado para la concepción de la interfaz. Es por eso que a Putnam le molesta que no entre en el Argumento por la Ilusión y todo eso (...) Él piensa que hago mi libro innecesariamente difícil presuponiendo trabajos anteriores sobre tales temas (Smith, 2002, p. 189) 7 .

\footnotetext{
${ }^{6}$ Las objeciones y réplicas a éstas de McDowell se registran en unas actas de la APA que el propio filósofo vuelve a recordar a Putnam en respuesta a su insistencia. En resumen: en tanto que Putnam dos veces objeta a McDowell, éste en ambas oportunidades reitera su disconformidad con que se asocie el empirismo mínimo al realismo ingenuo.

7 Traducción del autor del presente artículo.
} 
Si bien las teorías de los datos sensoriales caen bajo la crítica de McDowell, nunca en su defensa del empirismo mínimo discute a favor de la tesis del realismo directo. No lo hace, en efecto, al plantear el conceptualismo. Sin embargo, más allá de su propia ponderación en torno de la estructura de su obra, hay elementos en Mind and World para argumentar a favor de un realismo ingenuo frente a la objeción clásica que propone el argumento por la ilusión. Esos elementos no son engranajes sueltos en el desarrollo teórico de McDowell, sino que influyen -como veremos- en su defensa del carácter conceptual de la experiencia, tal vez mucho más de lo que el filósofo reconoce hasta el momento.

De todas formas cabe aclarar que nuestro artículo parte de una consideración de Putnam en torno a la conveniencia del vínculo entre empirismo mínimo y realismo directo. Sin embargo, no asume otros aspectos de la interpretación de Putnam por lo siguiente: Putnam no otorga relevancia a la discusión en torno a la naturaleza del contenido empírico. En La trenza de tres cabos, donde argumenta a favor de un realismo directo, la discusión en torno a la naturaleza del contenido empírico ocupa sólo cuatro páginas de más de doscientas (Putnam, 1997, pp. 181-185).

\section{El empirismo mínimo y el conceptualismo}

El empirismo mínimo de McDowell es relevante, entre otras razones, porque se opone a abordajes muy influyentes dentro de la epistemología contemporánea. Por una parte, al considerar que las experiencias pueden funcionar como razones para las creencias perceptivas, se opone al coherentismo epistemológico -cuyo representante característico es Donald Davidson (1983)-, conforme al cual sólo una creencia puede justificar a otra creencia. Para Davidson el vínculo entre pensamiento y mundo es estrictamente causal, esto es, las experiencias causan las creencias, pero no las justifican. Por el contrario, desde la perspectiva de McDowell el pensamiento pierde todo sentido sin una restricción externa de rango epistemológico, provista, en efecto, por la propia experiencia. Sin dicha restricción se corre el riesgo de dejar al pensamiento como un "engranaje girando en el vacío", que es la situación en la que justamente deja la relación entre la mente y el mundo el coherentismo davidsoniano (McDowell, 1994/6, p. 11).

Por otro lado, McDowell se enfrenta a aquellas perspectivas que asumen un dualismo tajante entre mente y mundo, logrando extender el espacio 
de la justificación más allá del espacio lógico de las razones. Siguiendo a Wilfrid Sellars, esta pretensión de postular entidades extra epistémicas como fundamento de entidades epistémicas - como son los juicios- cae en el 'Mito de lo Dado' (Sellars, 1997). Lo 'mítico' aquí es la incoherencia entre la caracterización de la experiencia como ajena al ámbito de las razones y la del pensamiento como algo esencialmente atado al ámbito de las razones, junto con la reivindicación de un vínculo de justificación que reuniría a una y otro. Sobre este trasfondo, el abordaje de McDowell busca dar con un concepto sustentable de experiencia, evitando tanto el coherentismo epistemológico como cualquier recaída en el 'Mito de lo Dado' (McDowell, 1994/6, p. 13).

En esta trayectoria un elemento fundamental para defender el empirismo mínimo es el conceptualismo. Basándose en la idea kantiana de que el pensamiento es un logro conjunto entre la espontaneidad del entendimiento y la receptividad de la sensibilidad, McDowell considera que los conceptos precisan de intuiciones sensibles y éstas de los conceptos (pp. 40-45). A su vez, indica que en el propio Kant se encuentra la tesis de que las contribuciones tanto de la espontaneidad como de la receptividad no son separables, sino que nuestras capacidades conceptuales ya están actuando pasivamente en la experiencia, y de forma activa en los juicios. Esta articulación o dependencia es funcional a la idea, por último, de que la experiencia funciona como razón, pues si la experiencia, a diferencia de lo que el empirismo clásico pensaba, participa del espacio lógico de las razones, puede entonces formar parte del proceso de justificación de nuestras creencias empíricas, sin recaer precisamente en el 'Mito de lo Dado':

Las capacidades conceptuales -afirma McDowell- se emplean en la receptividad (de la experiencia). (...) No es que se ejerzan en una entrega extra-conceptual de receptividad. Debemos entender lo que Kant llama "intuición" - una entrada experiencial- no como la obtención desnuda de algo Dado extra-conceptual, sino como un tipo de ocurrencia o estado que ya tiene contenido conceptual (...) en la experiencia uno capta (takes in), ve, por ejemplo, que las cosas son así y asá. Es el tipo de cosa que uno puede, por ejemplo, juzgar (p. 9) ${ }^{8}$.

8 Traducción del autor del presente artículo. 
Una característica fundamental del conceptualismo de McDowell es, entonces, su motivación epistemológica. Al igual que otros conceptualistas (Brewer, 1999; 2005), parte de la premisa de que la experiencia justifica los juicios y concluye que, de ser así, toda percepción debe tener naturaleza conceptual. La transición desde una premisa anti coherentista -la experiencia justifica los juicios- a la afirmación del conceptualismo, requiere extender el espacio lógico de las razones hasta incluir la experiencia.

Aunque un desafío importante, en este cuadro de posiciones epistemológicas, es explicar las críticas tanto al coherentismo como al 'Mito de lo Dado', la idea de que la experiencia pueda ser una fuente de conocimiento directo del mundo no resulta difícil de aceptar, solo debido a que el coherentismo ofrece una visión alternativa de la justificación, o debido a las críticas al 'Mito de lo Dado'. Aún antes de constatar que estas posiciones epistemológicas tienen cada una su punto de razón, hay ciertos aspectos fenomenológicos de la percepción que siempre han jugado un rol central en la reflexión filosófica. Precisamente la idea de que la experiencia es una fuente directa de conocimiento del mundo, es una preconcepción compartida ordinariamente en nuestra vida corriente. En esta medida, dar cuenta de esta característica resulta pertinente para un enfoque sobre la naturaleza del contenido perceptivo. Ahora bien, buena parte del pulso filosófico a partir de la Modernidad ha consistido en desestimar esta preconcepción mediante un poderoso y extendido argumento escéptico, referido a la posibilidad de que la experiencia sea falible. Discutir sobre la falibilidad de la percepción es un locus recurrente en la reflexión epistemológica desde el siglo XVII, debido a la injerencia de la tradición escéptica, a su vez ocasionada por el descubrimiento del texto del escepticismo griego Esbozos Pirrónicos (Popkin, 2003).

McDowell, si bien atiende a la intuición ordinaria de que la experiencia conoce directamente el mundo, no brinda relevancia a la falibilidad de la percepción como objeción a su postura. Esto se debe a que en su defensa del empirismo mínimo no discute el argumento por la ilusión que usualmente convenció a los empiristas clásicos de abandonar el realismo ingenuo. Entendemos que tomar en cuenta estas consideraciones y, por tanto, relacionar el conceptualismo con el realismo ingenuo, o directo, para enfrentar el argumento por la ilusión, no va en contra de la estrategia argumentativa de McDowell ni de lo "difícil" de su libro, sino que contribuye a aclarar ciertos aspectos en lo que respecta a la motivación epistemológica del conceptualismo. 


\section{La carencia de límites de lo conceptual}

\section{La objeción de idealismo}

El conceptualismo, es decir, la tesis de que la experiencia posee contenido conceptual "nos pone en posición de hablar", según McDowell (1994/6), “de la experiencia como la apertura a la estructura de la realidad" o sencillamente como "apertura al mundo" (p. 26). Esta idea de apertura a la realidad significa que es posible que el contenido de nuestra experiencia se identifique con hechos del mundo. Aun cuando el propio McDowell (2008) ha discutido aspectos técnicos de esta idea-tales como si la percepción tiene o no carácter proposicional- el caso es que implica que la experiencia tiene capacidad de representar conceptualmente aspectos del mundo de manera independiente a su ratificación en los juicios empíricos. Esto no quita que como buen empirista su interés esté en argumentar que la experiencia fundamenta nuestras creencias empíricas. Pero para que esto ocurra es necesario atribuir a la percepción la capacidad de ser una fuente de conocimiento.

Luego de presentar los escollos para su empirismo mínimo, McDowell explica en el segundo capítulo de su obra principal la tesis de "la carencia de límites de lo conceptual" (1994/6, pp. 25-29). Según su propia advertencia, ésta no da respuesta a los cuestionamientos más fuertes al empirismo mínimo, sino que pone de relieve con mayor claridad su posicionamiento al abordar una objeción "en la línea del idealismo" (p. 26). En otras palabras, en su propia valoración esta idea permitirá evitar objeciones menores, podríamos decir, sin atacar el grueso de la discusión. En contraste con su perspectiva, nuestra interpretación otorgará mayor importancia a este planteamiento.

La objeción es la siguiente: si la experiencia tiene contenido conceptual, entonces no habría nada externo al pensamiento. Todo el pensamiento se reduciría al ejercicio de nuestra capacidad de emplear conceptos. El idealismo tiene descrédito al ser considerado una trampa en donde finalmente los agentes quedan atrapados en el armazón de sus propias creencias o capacidades para conocer. Si bien no hay un sentido único de ser idealista, es claro que, en el contexto de la obra de McDowell, el idealismo se presenta como un punto negativo. "Una postura de idealista -afirma McDowellequivale a protestar porque no reconoce de modo genuino que la realidad es independiente de nuestro pensamiento. Si esto fuese correcto, mi afirmación de la independencia de la realidad seria poco sincera, meramente de boquilla" (p. 26). 
Es interesante que McDowell vincule la "acusación de idealismo" con "un antropocentrismo bien arrogante", que sostiene que el mundo es totalmente conocible desde el punto de vista humano tal como si se tratara de una imposibilidad de aceptar limitaciones para nuestra capacidad epistémica (pp. 39-40). Si bien esta objeción es buena, no tiene mayor desarrollo en el filósofo de Pittsburgh. Se aproxima a lo que en los últimos años se discute bajo el nombre de 'misterianismo'. Según el "misterianismo" hay problemas que son y serán incognoscibles por nuestra condición humana aun cuando tendamos a plantearnos dichos misterios (De Caro, 2019). Posiblemente hay aquí una vía de argumentación complementaria a la defensa de la carencia de límites de lo conceptual por parte de McDowell. Detenernos en esta complementariedad, sin embargo, excede el alcance de este artículo.

McDowell interpreta esta objeción de idealismo como "un reflejo de la convicción" (1994/6, p. 26) de que sólo existen el par de opciones que son su punto de partida, a saber, el coherentismo y el 'Mito de lo Dado'. En respuesta a ello, de todas formas, apela a la distinción entre "pensamiento y lo pensable", siendo el pensamiento la capacidad activa de realizar juicios, y lo pensable la receptividad pasiva de contenidos de la experiencia (pp. 27-29). En este tramo de la argumentación resulta difícil capturar esta diferenciación, que remite al Tractatus lógico-philosophicus (1921) de Wittgenstein. No obstante, cabe exponerla mediante un ejemplo.

Supongamos que yo creo que todos los automóviles de la ciudad son verdes. He elaborado este juicio en mi experiencia viviendo en el lugar y prestando atención constantemente al color de los automóviles. No es una creencia demasiado relevante en mi vida, pero es algo que tengo presente cada vez que salgo con el coche. Mi pensamiento es ese: que todos los autos son verdes en la ciudad. Sin embargo, un día cae bajo mi percepción un automóvil de color azul. La posibilidad de percibir exige desde ya que yo tenga cierta ejercitación en los colores, pero el caso es que no forma parte de mi voluntad o mi decisión que el coche sea de ese color azul. Lo pensable, en este caso, es una 'restricción' que me informa que hay al menos un automóvil azul en la ciudad porque lo veo. Por más que mi pensamiento sea que todos los automóviles son verdes, lo pensable me restringe a no mantener esa creencia. En tanto que mi pensamiento puede - y en este caso- debería modificarse, lo pensable es una suerte de dato para nuestro conocimiento:

9 Traducción del autor del presente artículo. 
El hecho de que la experiencia sea pasiva (que sea un caso en que la receptividad ha entrado en acción) debería cerciorarnos de que disponemos de toda la constricción externa que podemos razonablemente desear. La constricción nos llega desde fuera del pensamiento, pero no desde fuera de lo que es pensable. Cuando nos remontamos hasta el final de una justificación, lo último a lo que llegamos es, todavía, un contenido pensable: no algo más fundamental que eso (McDowell, 1994/6, p. 71).

Ahora bien, la objeción idealista, aun cuando apunta a cuestionar en términos generales la posibilidad de conocer más allá de las propias creencias, tiene una particular eficacia cuando se trata de la percepción. Esto se relaciona paradójicamente con el carácter intuitivo que a menudo adopta el idealismo como objeción. Si bien nadie duda frecuentemente de lo que ve, también es cierto que la eficacia del argumento por la ilusión coadyuva a su aceptabilidad. En tal sentido, cabe preguntar ¿de dónde habitualmente nos podría surgir la idea de que la experiencia no puede ser una fuente fiable de conocimiento? En el plano de la discusión filosófica Descartes ya nos da un indicio claro de esto al decir: “(...) he experimentado a veces que (los) sentidos me engañaban, y es prudente no fiarse nunca por entero de quienes nos han engañado una vez" (1977, p. 18). La expresión 'por entero' (plane) es una restricción importante del verbo 'confiar'. El mensaje de Descartes es claro: nunca confiar del todo (nunquam confidere plane illis -en alusión a los sentidos-), a partir de la constatación de que a veces los sentidos nos engañan. Esta desconfianza plena considera que dado que a veces los sentidos nos engañan no podemos confiarnos nunca de ellos como fuente de conocimiento directa del mundo (p. 18).

En efecto, desde los tiempos del escepticismo griego existe la objeción en torno de cómo explicar el error para los casos en que la experiencia no nos muestre hechos, o -para decirlo en términos del agente- para casos en los que nos equivocamos acerca de lo que percibimos. Si la experiencia, en el ejercicio de capacidades conceptuales, nos abre al mundo -tal como sostiene McDowell- ¿cómo se explican entonces los casos de ilusión perceptiva de aquello donde nos parece que vemos algo cuando en realidad no está allí? ¿No es acaso un hecho evidente que la experiencia a veces no se corresponde con los hechos? ¿En ese sentido, no resultaría entonces una afirmación infundada atribuir 'apertura al mundo', a nuestra experiencia, cuando es posible que un caso de percibir en rigor no nos represente hechos? 


\section{Ilusión perceptiva y posibilidad ordinaria vs Posibilidad en principio}

Entendemos que una forma clara de comprender esta objeción es mediante un ejemplo de ilusión perceptiva. Es importante denominar 'ilusión perceptiva' a una generalidad de casos en donde nos pareció que veíamos algo muy claramente y en realidad estábamos equivocados. En tal sentido, nos limitamos a concebir las ilusiones con cierta generalidad, pero en ningún caso incluyendo lo que John Austin denominó 'delusions' en Sentido y percepción (1962). En su ataque a las teorías de los datos sensoriales Austin distinguía entre 'delusions' e 'ilusions', siendo las 'delusions' errores en donde estaban "comprometidas" las credenciales del agente (por ejemplo, alguien que ve elefantes rosas en la clase cuando de hecho todos sabemos que no existe tal cosa), e ilusiones en donde estas credenciales no están comprometidas como las del ejemplo o, más sencillas, como la ilusión o "ver" la barra quebrada bajo el agua (Austin, 1981). En las últimas décadas Fish (2009) ha profundizado esta distinción en relación con el argumento por la ilusión entre 'ilussion' y 'hallucination'.

Dicho esto, supongamos que voy a un recital y veo a mi cantante favorito. Supongamos además que, por ciertos excesos propios del rock, ese cantante no estuvo en condiciones de dar ese recital. Sin embargo, acostumbrados a estos desplantes, su grupo técnico desde hace tiempo ha diseñado un holograma que emula la imagen del cantante en el escenario. Precisamente la noche en que debo ir a su recital sucedió que el cantante no pudo asistir personalmente. Sin embargo, su holograma creó la ilusión en mí -y en todo el auditorio- de que efectivamente estaba allí en persona. Supongamos, además, que he percibido en condiciones subjetivas ordinarias, no distorsivas (no estaba bajo los efectos de las drogas). Además, estimemos, tenía buena visibilidad (estaba a 10 metros del escenario). Sin embargo, mi percepción no es verídica. Si esta posibilidad es perfectamente probable, aun cuando pueda ser excepcional e incluso exótica, ¿su mera constatación no debería limitar la convicción de que la experiencia nos abre al mundo tal como lo sostiene McDowell?

En relación a objeciones como éstas no hay elementos explícitos en Mind and World, ni en la obra subsiguiente, que contribuyan a morigerar esta suspicacia escéptica. Sin embargo, el filósofo africano considera, en el camino a su defensa del empirismo mínimo, la falibilidad de la percepción. Esto no impide, de todas maneras, que no le conceda toda su eficacia como objeción. Prueba de ello es que ni siquiera otorga estatuto teórico al argumento. Al explicar 
qué pasa en casos donde nos equivocamos, no obstante, afirma lo siguiente: "cuando reconocemos la posibilidad de equivocarnos, no eliminamos con ello la posibilidad de que «captar las cosas» sea una descripción de lo que ocurre cuando uno no se equivoca" (McDowell, 1994/6, p. 26).

La simpleza de esta afirmación encierra un elemento importante que años más tarde adquirirá una relevancia singular en la trayectoria de McDowell. Dicho en resumidas cuentas la idea es la siguiente: la equivocación es algo visible sobre un trasfondo de aciertos. En el ejemplo, sé que me he equivocado contrastando el caso en que efectivamente el cantante brinda su recital (la mayoría de las veces). A su vez si he accedido al recital con cierta expectativa es que efectivamente he tenido casos de percibir que han captado que las cosas son así y asá. De lo contrario, si tuviera razones para desconfiar todo el tiempo de cómo mi experiencia capta las cosas, no tendría expectativa alguna de tener un conocimiento fiable del mundo, ni por tanto tendría accesibilidad a la idea de 'no estar equivocado'. De este modo, si bien es cierto que mi experiencia del cantante estaba equivocada, si bien es cierto que algunas veces nos equivocamos respecto de cómo nuestra experiencia capta las cosas, dicha constatación ha sido posible porque en incontables veces he accedido al mundo a través de la experiencia. En otras palabras, cuando no ha sucedido que estuviera equivocado, efectivamente mi experiencia ha captado incuestionablemente rasgos del mundo. Atendiendo a esta probabilidad se puede afirmar de manera justificada que cuando estoy en lo cierto, la percepción me abre a rasgos del mundo.

En "Perception as a capacity for knowledge" McDowell (2011) desliza afirmaciones en una tónica similar. En este caso cree que en lugar de hablar de que la percepción 'conoce', es conveniente decir que 'estamos equivocados o no lo estamos cuando percibimos’. En este último caso la percepción se comprende como una capacidad que, en cuanto tal, a veces puede verse afectada por cuestiones del contexto. De allí que McDowell (p. 30) emplee la expresión "when all goes well" como una concesión necesaria previa a afirmar que la experiencia, en tanto capacidad, puede abrirnos al mundo: "Cuando todo va bien -afirma- en el funcionamiento de una capacidad perceptiva de un tipo que pertenece a la racionalidad de su poseedor, quien percibe disfruta de un estado perceptivo en el que alguna característica del entorno está allí para ella, presente perceptualmente en su conciencia racionalmente consciente de sí misma" (pp. 30-31). 
Cuando "todo va bien" quien percibe es consciente de un tipo de conocimiento que consiste en un rasgo del mundo. Podemos apelar incluso a dicho rasgo para fundamentar ciertos juicios si, eventualmente, se nos demanda que acreditemos nuestro conocimiento. Este lenguaje de capacidad debe comprenderse como un atributo fundamental del tipo de conocimiento empírico del que somos capaces los seres lingüísticos. En este ensayo, McDowell entiende que enfatizar este punto ayudará a despejar ciertos malos entendidos en torno a la falibilidad de la percepción y a la plausibilidad de que la experiencia ofrezca garantías de conocimiento. Sobre ambos puntos, por cierto, sostiene un debate con Tyler Burge, cuyo abordaje excede los límites del artículo:

La falibilidad es una propiedad de las capacidades, o quizás de los sujetos cognitivos como poseedores de capacidades. Si una capacidad es falible, o si, para hablar de esa otra manera, cualquiera que la tenga es falible con respecto a ella, eso significa que puede haber ejercicios de la capacidad en la que su poseedor no hace lo que la capacidad se especifica como una capacidad de hacer (p. 37).

La percepción es una capacidad que nos abre al mundo. Ahora bien, pudiera suceder que quienes ejecutan esa capacidad no logren el cometido. Al decir que cuando las cosas van bien ese cometido se logra, a lo sumo se implica que en la mayoría de los casos dicha capacidad debería funcionar. Sin embargo, esto no es suficiente porque lo más difícil de elucidar, en este punto, es ¿cuándo efectivamente no deberíamos dudar de que nuestra capacidad de experiencia nos ofrezca conocimiento empírico?

Los señalamientos de McDowell desde ya resultan desalentadores para un escéptico radical que permanece en su duda. Sin embargo, hay matices que resultan necesarios inclusive si no se llega tan lejos en el cuestionamiento. Se trata de matices que hacen posible que la conclusión de McDowell no sea una mera confirmación de la fenomenología de la percepción. Es aquí cuando entendemos que hay una distinción en los comentarios citados, en parte implícita y en parte no. Se trata de la distinción entre 'posibilidad fáctica u ordinaria' y 'posibilidad en principio' de error perceptivo.

Veamos. La posibilidad de que estemos en lo cierto como de que nos equivoquemos está limitada a la 'posibilidad fáctica' del descubrimiento del engaño. En el ejemplo del recital se puede descubrir la ilusión de la que fui víctima y se puede saber cuándo un holograma reemplazó al cantante. 
Podría, por ejemplo, presentarse evidencia al respecto; supongamos un informe periodístico que denuncie la estafa. Dado que, si no hubiera aciertos mayormente, en este sentido, yo no habría detectado el error, se sigue que es razonable la afirmación de que la experiencia nos abre al mundo. La conclusión no impide la posibilidad de que haya errores perceptivos (casos de ilusión). Ahora bien, esas ilusiones sólo son identificables sobre un trasfondo de percepción exitosa, de apertura al mundo. De modo que si no se acepta que la percepción acierta la mayoría de veces, tampoco puede reconocerse que a veces falla.

La conclusión de McDowell explota el sentido de posibilidad en términos de posibilidad fáctica. Sin embargo, no lo hace en los términos de una 'posibilidad de principio'. Veamos: en principio es posible que todas nuestras percepciones en conjunto sean producto de un engaño. Podría darse incluso que una gran matrix nos controlara desde un centro de operaciones y que nuestros cuerpos no sean más que cerebros en una cubeta, estimulados por descargas de diverso tipo para incitarnos representaciones sobre el mundo que son totalmente artificiales. O bien podría darse la hipótesis del genio maligno que puede hacernos convencer de que incluso juicios matemáticos erróneos son correctos tales como $3+3=5$. Apelando a objeciones de principio como éstas, el escéptico parece siempre permanecer en pie. Nótese que hablamos de objeciones en principio como independientes de la experiencia, ya que no habría posibilidad de presentar evidencia que disuada al cuestionamiento en ninguna circunstancia humanamente concebible.

Sin embargo, en términos de una posibilidad fáctica, entendida ésta como algo que ocurre en un contexto ordinario y algo de lo cual se puede presentar evidencia que confirme o disuada el sostenimiento de ciertas creencias empíricas - una posibilidad ordinaria-, su sola delimitación supone una sistematicidad de aciertos. Tomando en cuenta esto, la respuesta de McDowell a la objeción escéptica limita los alcances del escéptico al mostrar su poca incidencia en el mundo ordinario. En cambio, no rebate ni pretende rebatir al escéptico radical que se monta sobre la posibilidad de principio. Y eso le resulta suficiente a los fines de su empirismo mínimo.

$\mathrm{Si}$, por una parte, las capacidades conceptuales se emplean tanto en la experiencia, receptiva o pasivamente, como en el ejercicio de realizar juicios (aquí de manera activa), y si, por otro lado, la forma de calificar nuestros juicios sobre el mundo es apelando a la experiencia, se sigue que nunca accedemos a un ámbito extra-conceptual para ponderar o calificar nuestros juicios o 
creencias. Por consiguiente, tanto en los juicios como en la experiencia se ejerce el mismo género de actividad conceptual, aunque de manera diferente -en un caso activamente y en el otro de manera pasiva. Por ende, la relación entre lenguaje y mundo está mediada por nuestras capacidades conceptuales. Lo conceptual, entonces, no tiene límites; no hay un límite por fuera de lo conceptual que determine la práctica de justificar nuestros juicios empíricos. Nuestra experiencia, cuando no está equivocada, nos abre al mundo.

En resumidas cuentas, la tesis de la carencia de límites de lo conceptual sostiene que no hay un límite desde la experiencia que ubique a ésta fuera del ámbito de lo conceptual. Dado que usualmente la experiencia se considera 'el otro lado' del límite de la esfera de lo conceptual -el ámbito de nuestros juicios o creencias-, esto le daría autoridad para ejercer una restricción 'externa' al pensamiento. Pero en ausencia de tal limitación, lo conceptual es "ilimitado". Precisamente ya no hace falta extender el espacio de la justificación más allá del espacio de las razones para garantizar el rol epistémico de la experiencia. La experiencia es conceptual y esto, a su vez, no impide que preserve el carácter de estar condicionado por restricciones racionales que se nos imponen y a las cuales usualmente, salvo circunstancias excepcionales, damos entero crédito.

\section{Conclusiones}

Hemos vinculado la defensa del conceptualismo con la afirmación de un realismo ingenuo o directo. Putnam (1997) llama a esta variante "realismo natural" en alusión a la correspondencia con lo que naturalmente ajusta con nuestra fenomenología de la percepción (pp. 12-14). Siguiendo este mismo criterio la denomina asimismo "realismo de sentido común". Hemos ofrecido una reconstrucción posible del modo en que Mind and World enfrentaría la cuestión de la falibilidad de la percepción en la versión extrema que plantea el argumento por la ilusión. Para ello no hemos fijado como pretensión hablar en términos de lo que hubiera dicho McDowell. Es evidente que McDowell rechaza la relevancia de la defensa del realismo ingenuo para sostener su conceptualismo. Pero también demostramos que en Mind and World se hallan elementos para dar respuesta a la falibilidad de la percepción. En función de ello, hemos sostenido que la falibilidad al percibir se comprende en dos sentidos: como una posibilidad fáctica y como posibilidad en principio; de esta última se vale el escéptico radical. Reconocer así en tal sentido que a veces los sentidos nos engañan, no nos conduce -a diferencia de lo que Descartes pensaba- a que la experiencia no nos abre al mundo cuando no estamos equivocados. 
La idea de posibilidad de error debe interpretarse aquí como una posibilidad ordinaria o factual, entendida como aquella que puede ser rebatida mediante una evidencia determinada, como sucede en nuestro ejemplo del recital. Esta posibilidad se opone a la que opera en la objeción escéptica radical: una noción de posibilidad de principio que es insensible a cualquier constreñimiento racional y que, estamos en posición de afirmarlo ahora, es la que otorga su eficacia al argumento por la ilusión.

La idea de que lo conceptual es ilimitado permite explicar cómo el conceptualismo resuelve el tópico de la falibilidad de la percepción, en el marco de una defensa del empirismo mínimo. Hemos argumentado que esta noción malentendida es perniciosa. Hay particularmente un malentendido asociado a ella, que podemos ampliar en el marco de estas consideraciones finales.

Cierta característica de lo conceptual está relacionada con el control racional sobre los contenidos mentales. Si el modelo de actividad conceptual es la formulación de un juicio, un juicio supone deliberación y por tanto control racional, de quien lo realiza. Esto puede ser ridiculizado aplicado a la idea de que la experiencia puede ser la responsable de restringir nuestro pensamiento -tal como lo propone McDowell. Si la experiencia es conceptual y lo conceptual de alguna manera cae bajo el control racional de los agentes ¿esto querría decir que percibimos lo que se nos antoja o peor aún que el mundo es aquello que se les antoja a los agentes o que cae antojadizamente bajo su percepción? Hemos intentando mostrar que si no se insiste en una buena explicación en torno de la falibilidad de la percepción esta objeción de idealismo perniciosa o destructiva le cabe en parte a Mind and World -al menos en una lectura lineal de dicha obra.

Por otra parte, creemos que asumir la idea de la percepción como una 'capacidad' -tal como McDowell lo hace desde 2011-, permite hablar más claramente de las limitaciones de nuestro modo de conocer a través de la experiencia. No obstante, esta manera de referir a la experiencia debería habilitar, sin embargo, el desarrollo de una mayor complejidad para hablar de los casos de yerro perceptivo. En McDowell no encontramos todavía algo similar a esta estrategia.

Putnam, muy estimulado por las ideas de McDowell, abogó por una concepción "transaccionalista" de la percepción, en la que los casos de yerro perceptivo se explicarían debido a una mezcla de factores repartidos entre el 
individuo y la influencia del entorno (Putnam, 2012). En cuanto a McDowell, en sus últimas intervenciones sigue discutiendo la capacidad de la percepción para restringirnos racionalmente (2018). Allí insiste en que la experiencia puede ofrecer un fundamento "incorregible" para nuestros juicios. Dicha incorregibilidad no puede ser entendida sin distinguir la posibilidad de error tal como hemos hecho. El escepticismo, en su variante extrema o radical, puede ofrecer casos en donde evidentemente la garantía ofrecida por la experiencia no es incorregible.

Despejando el obstáculo escéptico, sin embargo, la gran amenaza para el posicionamiento de McDowell es el externalismo epistemológico, según el cual puede haber garantías a nuestro conocimiento que sean externas a la conciencia del agente. Si bien el escéptico montado en la duda radical está destinado a permanecer en pie, paulatinamente la falibilidad de la percepción empieza a parecer como una sombra acechante en la órbita de intereses del filósofo de Pittsburgh, aún bajo el ropaje de esta objeción externalista. Desde ya que esta interpretación 'escéptica' sobre el externalismo merece un ensayo aparte. De todas maneras, entendemos que esta tarea de explicitar algunos elementos implícitos en las complejas argumentaciones puede corresponderse con otras partes de Mind and World así como a trabajos posteriores. Esto evidencia el valor de una obra en el sentido más estimulante y fructífero de la palabra.

\section{Referencias}

Austin, J. (1981). Sentido y percepción. Barcelona: Tecnos.

Brewer, B. (1999). Perception and reason. Oxford: Oxford University Press.

Brewer, B. (2005). Perceptual experience has conceptual content. E. Sosa and M. Steup (Eds.), Contemporary Debates in Epistemology (pp. 217 230). London: Blackwell.

De Caro, M. (2019). The indespensability of the manifest image. Philosophy and social criticism, 20 (10), pp. 1-11. https://doi. org/10.1177/0191453719826615

Descartes, R. (1977). Meditaciones metafisicas. Con objeciones y respuestas (F. Vidal Peña, Trad.). Madrid: Alfaguara. 
Davidson, D. (1983). A coherence theory of truth and interpretation. E. Lepore and K. Ludwig (Eds.), The essential Davidson (pp. 225-237). Oxford: Oxford University Press.

Fish, W. (2009). Perception, hallucination and illusion. Oxford: Oxford University Press.

Martin, M. (2002). The transparency of the experience. Mind \& Language, 17 (4), pp. 376-425. https://doi.org/10.1111/1468-0017.00205

McDowell, J. (1994/6). Mind and World. Cambridge Mass.: Harvard University Press.

McDowell, J. (1998). Meaning, Knowledge and Reality. Cambridge Mass.: Harvard University Press.

McDowell, J. (2003). Mente y mundo (M. Á. Quintana Paz, Trad.). Salamanca: Sígueme.

McDowell, J. (2008). Avoiding the Mith of The Given. Having the World in View. Essays on Kant, Hegel and Sellars (pp. 256-265). Harvard: Harvard University Press.

McDowell, J. (2011). Perception as a capacity for knowledge. Milwaukee: Marquette University Press.

McDowell, J.(2018). Perceptual experience and empirical rationality. Analytic Philosophy, 59 (1), pp. 89-98. https://doi.org/10.1111/phib.12120

Popkin, R. (2003). The history of skepticism. From Savonarola to Bayle. Oxford: Oxford University Press.

Putnam, H. (1997). La trenza de tres cabos. Mente, mundo y acción. Barcelona: Siglo XXI.

Putnam, H. (2012). La filosofia nell' età della scienza. Bologna: Il mulino.

Sellars, W. (1997). Empiricism and the philosophy of mind. Cambridge Mass.: Harvard University Press.

Smith, N. (Ed.). (2002). Reading McDowell. On Mind and World. London: Routledge. 